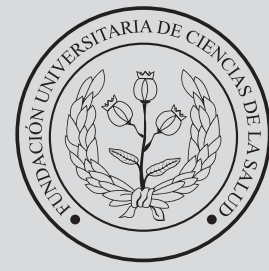

FUCS
Re per

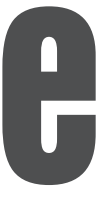

\title{
Los métodos educativos hacia un desarrollo de habilidades y aprendizaje imprescindibles
}

July Bibiana Castro $\mathrm{MD}^{\mathrm{a}}$ Nicolás Andrés Zambrano ${ }^{b}$ Valeria Andrea Villanueva ${ }^{b}$ Cristian Yecid Goyeneche ${ }^{b}$

\section{Educational methods towards developing indispensable}

skills and knowledge

a Medicina general, Fundación Universitaria de Ciencias de la Salud. Bogotá DC, Colombia.

${ }^{b}$ Facultad de Medicina, Fundación Universitaria de Ciencias de la Salud. Bogotá DC, Colombia.

\section{R E S U M E N}

La educación es un área que requiere constante actualización e innovación, tanto en la información transmitida, como en la metodología utilizada para poder desarrollar un proceso de enseñanza-aprendizaje adecuado, por lo que se deben usar diferentes estrategias que se acomoden a las necesidades y llamen la atención de los estudiantes. Dentro del área de la salud, es claro que uno de los objetivos primordiales del proceso educativo es la plena trasmisión de información, la consolidación de conceptos y la adquisición de destrezas por parte del estudiante, pero con el paso del tiempo los métodos convencionales han perdido validez y demuestran menor calidad con respecto a nuevas estrategias pedagógicas que se han implementado.

Las tecnologías de la información y la comunicación (blogs, cursos en línea, podcasts, entre otros), desempeñan un papel relevante dentro del proceso de actualización en métodos educativos, ya que son herramientas más asequibles y modernas y que pueden diseñarse de manera concreta y atractiva para los estudiantes, por lo que son de suma importancia tenerse en cuenta a la hora de diseñar programas pedagógicos y aplicarlos a situaciones de contingencia, como la que se vive actualmente.

Palabras clave: educación, pedagogía, blog, aprendizaje, medicina.

(C) 2020 Fundación Universitaria de Ciencias de la Salud - FUCS Este es un artículo Open Access bajo la licencia CC BY-NC-ND (http://creativecommons.org/licenses/by-nc-nd/4.0/).

\section{INFORMACIÓN DEL ARTÍCULO}

Historia del artículo:

Fecha recibido: julio 6 de 2020

Fecha aceptado: julio 24 de 2020
Autor para correspondencia.

July Bibiana Castro

jbcastro@fucsalud.edu.co
DOI

10.31260/RepertMedCir.01217372.1099 
The field of education requires continuous updating and innovation, both of the information transmitted and the methodology used, in order to develop an appropriate teaching-learning process. Therefore, different strategies must be used to adapt to the needs of the students and attract their attention. In the area of health sciences, it is clear that one of the primary objectives of the educational process is the full transmission of information, the consolidation of concepts and the acquisition of skills by the student, nevertheless, over time, conventional methods have lost validity and demonstrate lower quality compared to new pedagogical strategies that have been implemented.

The Information and Communication Technologies (blogs, online courses, podcasts, among others), play a relevant role within the process of updating educational methods, since they are more affordable and modern tools that can be designed in a concrete and attractive manner for students, so it is of utmost importance to take them into account when designing pedagogical programs and applying them to contingency situations, such as the one we are currently experiencing.

Key words: education, pedagogy, blog, learning, medicine.

(C) 2020 Fundación Universitaria de Ciencias de la Salud - FUCS. This is an open access article under the CC BY-NC-ND license (http://creativecommons.org/licenses/by-nc-nd/4.0/).

\section{INTRODUCCIÓN}

El área de la educación ha evolucionado, se han sumado herramientas que además de facilitar el aprendizaje, amplían la forma de adquirir conocimiento manteniendo diferentes flujos de información individual que permiten mantener a la población estudiantil mucho más en contacto con la academia, y así mismo aumentando la demanda de la misma. Estos métodos resaltan aún más en la situación actual a la que nos ha llevado la pandemia por COVID-19, sabiendo que lo que antes era una opción ahora es una necesidad para alcanzar un adecuado proceso pedagógico y un desempeño académico ideal. Las ventajas de esta modalidad de educación se evidencian cada vez más, estudios y reportes indican el efecto positivo y la respuesta satisfactoria que las nuevas herramientas han brindado a nivel académico.

Teniendo en cuenta que la aplicación de métodos innovadores para la educación puede llegar a acarrear algunos inconvenientes como lo es un aprendizaje poco sustancial, es importante resaltar que la evidencia y bibliografía citada en este artículo, apoya por encima de los modelos de enseñanza antiguos, la implementación de tecnología y modelos alternativos han generado un impacto importante en la integración y percepción de la información brindada, llevando a que se adquiera el conocimiento de forma más sencilla y garantizando que el mismo sea perpetuo. Es así como se ha abierto un nuevo campo de posibilidades que de una manera más didáctica, se adapta a la tecnología y a la nueva era. Como se ha evidenciado en varios estudios, estos avances llevarán a la población estudiantil a otro nivel académico, en el que las fallas a nivel del sistema educativo irán en decrescendo, mejorando la calidad de vida individual, estudiantil y eventualmente la formación de profesionales.

\section{MARCO TEÓRICO}

A medida que pasan los años la educación ha evolucionado hacia un aprendizaje auto dirigido, por el incremento de variedad de herramientas y plataformas con las que disponemos hoy en día, así como lo son los cursos en línea, herramientas online, cursos asincrónicos, entre otros. Lo anterior conlleva a tener diversas técnicas a nuestra disposición, y así mismo que sean rápidas para utilizar como por ejemplo lo es el "bloguear", las clases invertidas, las herramientas digitales y demás; se han visto cambios sobre cómo la información es presentada, llevando a un aprendizaje fuera del salón de clase, ya que el internet se ha convertido en un flujo de información individual, al que se tiene acceso desde donde sea y a cualquier hora, esto ha llevado a que la comercialización de información de cultura general y material educativo haya aumentado el interés y la demanda por estas fuentes de conocimiento. ${ }^{1}$

Pero existe un reto para todos los usuarios y así mismo para aquellos autores que administran estas páginas, y es el de no convertir el aprendizaje en algo superficial por la sobrecarga de información, omitiendo prestar atención al contenido de lo que se lee, por ello es de suma importancia la responsabilidad que se tiene sobre el contenido y la forma en que se da a conocer la información, para no solo tener un aprendizaje de calidad, sino también que se brinde de forma didáctica y entretenida para el lector; y que no se convierta en una retahíla que puede llegar a ser exhaustiva y monótona. ${ }^{1}$

Un estudio comparativo realizado en la Escuela de Medicina de Harvard, Boston, Massachusetts por Juan E. Small (Md.,Partners Healthcare) implementó un nuevo modelo pedagógico en un grupo de estudiantes de medicina que adicionalmente estuviesen cursando la asignatura de 
anatomía. Implementaron módulos virtuales de aprendizaje mediante el uso de imágenes radiológicas, para así reemplazar el modelo tradicional que se iba manejando; esto con el fin de identificar el nivel de desarrollo académico y de satisfacción de este grupo de estudiantes. Lograron concluir que el uso de tecnologías de información dentro de modelos de aprendizaje actuales, permite que la información sea aprovechada de mejor manera dentro del transcurso de cada sesión de clase y que los estudiantes mantengan un nivel de motivación más alto frente a la asignatura. Adicionalmente, evidenciaron que la integración de información tradicional con el diseño de nuevos modelos virtuales didácticos muestra gran acogida por la población académica, por encima de los modelos de enseñanza antiguos. ${ }^{2}$

Así mismo, en un estudio de cohortes en Grenoble, Francia realizado por Pierre Gallois y col. buscaban actualizar un modelo de enseñanza que manejaba clases de grupos grandes y que resultaban poco eficientes, a partir de un sistema de aulas invertidas con el que se permitiera mejorar el nivel de éxito de los estudiantes de medicina de primer año, poniendo en evidencia los factores externos que realmente influyen dentro de los resultados de los estudiantes en el examen para acceder a segundo año. Los autores lograron observar que el nuevo modelo permitió aumentar la entrada de estudiantes a segundo año y de la misma manera identificar los verdaderos factores que realmente influyen dentro de los resultados del examen final de los estudiantes (el tener una mención de honor por el examen final de la secundaria y que el estudiante haya repetido el año); por lo que la implementación de este innovador modelo, permitió que los factores socioeconómicos pasen a un segundo plano y las oportunidades de ingresar al siguiente año sean más equitativas entre todos los estudiantes. ${ }^{3}$

Abreu, Judith, Curbeira, Eduardo y col. en su artículo de opinión acerca de que es un blog y como el avance tecnológico y su alcance ha llegado de forma más fácil a la comunidad, aparte de resaltar sus ventajas acerca de la interacción del autor con sus lectores a través de comentarios y actividades que pueden ser manejadas de forma muy práctica por el dueño del blog, relaciona algunos ejemplos de diversos blogs de ciencias de la salud. El artículo da algunas técnicas acerca de la integración entre otras páginas que puedan estar relacionadas. Convierten el blog desde su base conceptual de diario web a una comunidad de información ajustada al lector. Así mismo llegan a la conclusión de que el uso de blogs es bastante amplio y el potencial que tienen con el avance de la tecnología, permitirá una mejora en el proceso educativo y un alcance superior en la comunicación. ${ }^{4}$

De igual manera Reynaldo Badillo, en su artículo de revisión publicado de la Revista Salud Uninorte de Bucaramanga, busca enseñarle a la comunidad, en principio, la evolución de la web desde su versión 1.0 y la 2.0, además del alcance y las nuevas interacciones que existen entre los actores de este tipo de comunicación tecnológica. Además, no solo se habla de blogs sino también de podcasts; Wikis y otras aplicaciones que pueden tener enlaces e interactividad amigable entre el autor y sus lectores. De igual forma, relata el uso de RSS que permite una comunicación y aviso directo a los lectores de acuerdo a sus intereses y suscripciones, teniendo en cuenta que en comunidades jóvenes estos medios de comunicación han tenido gran impacto y han permitido la difusión del conocimiento de una forma resumida, práctica y entendible, por lo cual lograron deducir, que la Web 2.0 es la evolución del internet donde la comunicación es bidireccional entre el lector y autor, además es un nuevo método de servicios con mayor eficacia, dinamismo y funcionalidad, tanto en la sociedad como en la educación médica. ${ }^{5}$

Dicho esto, es de suma importancia resaltar las nuevas estrategias de educación virtual, puesto que han desempeñado un papel clave dentro de la pandemia que se vive actualmente, ya que más que ser una ventaja, pasa a ser una necesidad dentro del proceso educativo. Esta situación demanda un uso exclusivo de estas herramientas, llevando a que la enseñanza sea más personalizada al momento de transmitir información, resolver dudas y al desarrollo de hábitos hacia un estudio autodirigido. Se debe tener claro que este es el momento para que la humanidad las aplique como herramientas dentro del proceso pedagógico como por ejemplo temas de salud-enfermedad, entre otros, y así usar las tecnologías de la información y la comunicación para lograr una mejor enseñanza. ${ }^{6}$

\section{CONCLUSIÓN}

- La educación y los métodos utilizados han evolucionado en el tiempo, de acuerdo con la necesidad y los avances tecnológicos de la actualidad, por lo cual se han convertido en herramientas adaptables, versátiles, accesibles e interactivas, utilizadas a nivel multidisciplinario.

- La educación ya no está limitada a un salón físico y a la presencialidad, la tecnología ha generado conexiones e instrumentos para salir de estos espacios, y crear una modalidad modernizada, la cual se nutre día a día de los avances de las ciencias, con disminución en tiempos de comunicación y ampliación de los canales de recepción de información.

- Los nuevos métodos de aprendizaje, como el uso de blogs, brinda espacios con contenido de calidad enfocado en el área requerida, con material pedagógico basado en la evidencia actual y presentada de forma práctica para facilitar el aprendizaje y la adquisición de conocimiento.

- Ya sea desde grandes o pequeños niveles, la actual situación de la pandemia por COVID-19 nos ha demostrado que podemos generar y recibir aprendizaje, con múltiples plataformas y medios pedagógicos, pero así mismo la información siempre ha de ser corroborada, basada en evidencia y bibliografía adicional. 


\section{AGRADECIMIENTOS}

El presente artículo fue realizado bajo la supervisión de la doctora Karen Muñoz, médica especialista en medicina familiar y coordinadora del semillero de investigación de medicina familiar y comunitaria de la Fundación Universitaria de Ciencias de la Salud a quien presentamos nuestros más profundos agradecimientos por su paciencia, tiempo y dedicación; así mismo por compartir sus conocimientos y guía para lograr realizar este trabajo.

\section{CONFLICTO DE INTERÉS}

Los autores declaran no tener ningún conflicto de intereses.

\section{REFERENCIAS}

1. Thaung C. Blogging for medical education - a personal view. J R Coll Physicians Edinb. 2018;48:48-9. doi: 10.4997/JRCPE.2018.112

2. Shaffer K, Small JE. Blended learning in medical education: use of an integrated approach with web-based small group modules and didactic instruction for teaching radiologic anatomy. Acad Radiol. 2004;11(9):1059-70. doi: 10.1016/j.acra.2004.05.018

3. Gillois P, Bosson JL, Genty C, Vuillez JP, Romanet JP. The impacts of blended learning design in first year medical studies. Stud Health Technol Inform. 2015;210:607-11.

4. Martínez Abreu J, Curbeira Hernández E. El blog, una herramienta de la web para potenciar el proceso docente educativo en las carreras de las Ciencias Médicas. Rev Med Electrón. 2014;36(3):381-7.

5. Badillo Abril R. Aplicaciones y estrategias "Web 2.0" en la Educación Médica. Revista Salud Uninorte. 2011;27(2):275-88.

6. Sahi PK, Mishra D, Singh T. Medical Education Amid the COVID-19 Pandemic. Indian Pediatr. 2020. 